\title{
Impact of NRAS Mutations on the Diagnosis of Follicular Neoplasm of the Thyroid
}

\author{
Ja-Seong Bae, ${ }^{1}$ Seung Kyu Choi, ${ }^{2,3}$ Sora Jeon, ${ }^{2}$ Yourha Kim, ${ }^{2}$ Sohee Lee, ${ }^{1}$ \\ Youn Soo Lee, ${ }^{2}$ and Chan Kwon Jung ${ }^{2}$ \\ ${ }^{1}$ Department of Surgery, College of Medicine, The Catholic University of Korea, Seoul 137-701, Republic of Korea \\ ${ }^{2}$ Department of Hospital Pathology, College of Medicine, The Catholic University of Korea, 222 Banpodaero, Seocho-gu, \\ Seoul 137-701, Republic of Korea \\ ${ }^{3}$ Department of Pathology, College of Medicine, Dankook University, Cheonan-si, Chungnam 330-714, Republic of Korea
}

Correspondence should be addressed to Chan Kwon Jung; ckjung@catholic.ac.kr

Received 3 July 2014; Revised 18 August 2014; Accepted 20 August 2014; Published 31 August 2014

Academic Editor: Sabrina Corbetta

Copyright (C) 2014 Ja-Seong Bae et al. This is an open access article distributed under the Creative Commons Attribution License, which permits unrestricted use, distribution, and reproduction in any medium, provided the original work is properly cited.

\begin{abstract}
Background. Most patients with a preoperative diagnosis of thyroid follicular neoplasm (FN) undergo diagnostic surgery to determine whether the nodule is benign or malignant. Point mutations at NRAS codon 61 are the most common mutations observed in FN. However, the clinical significance of NRAS mutation remains unclear. Methods. From 2012 to 2013, 123 consecutive patients undergoing thyroidectomy for FN were evaluated prospectively. Molecular analyses for NRAS codon 61 were performed with pyrosequencing. Results. The overall malignancy rate in FN was $48.8 \%(60 / 123)$. Of 123 FNs, $33(26.8 \%)$ were positive for the NRAS mutation. The sensitivity, specificity, positive predictive value, and negative predictive value of a NRAS mutation-positive FN specimen to predict malignancy were $37 \%, 83 \%, 67 \%$, and 58\%, respectively. Patients with a NRAS-positive FN had a higher malignancy rate in additional thyroid nodules beyond the FN than patients with a NRAS-negative FN. The overall malignancy rate of patients with a NRAS-positive FN was significantly higher than that of patients with a NRAS-negative FN $(79 \%$ versus $52 \%$; $P=$ 0.008). Conclusions. Determining NRAS mutation status in FN helps to improve the accuracy of thyroid cancer diagnosis and to predict cancer risk in accompanying thyroid nodules.
\end{abstract}

\section{Introduction}

A follicular neoplasm (FN) is a thyroid nodule that can be cancerous or noncancerous, a follicular carcinoma or a follicular adenoma, respectively. The rate of malignancy is $20-30 \%$ in nodules with FN $[1,2]$. The definitive morphological diagnosis of follicular thyroid carcinoma (FTC) relies on a demonstration of capsular or vascular invasion after surgery. Many patients with follicular adenoma are operated on unnecessarily because it is not possible to discriminate between benign and malignant FN with a preoperative fine needle aspiration biopsy (FNAB) or a core needle biopsy (CNB) [3].

In recent years, molecular-based diagnostic markers, such as BRAF, RAS, PAX8-PPAR $\gamma$, or RET-PTC mutations, have improved the diagnosis of thyroid nodules [4]. The $B R A F$ V600E mutation is the most common genetic alteration in thyroid tumorigenesis and has been observed in $\sim 29-83 \%$ of papillary thyroid carcinoma (PTC) [5-7]. RAS mutations are the second most common genetic alteration in thyroid tumors. Recent studies reported that $10-20 \%$ of PTC and $40-50 \%$ of FTC harbor RAS mutations [8,9]. They have been associated with poor prognoses and tumor dedifferentiation $[10,11]$. The RAS genes consist of three families: NRAS, HRAS, and KRAS. RAS point mutations mostly occur in codons 12,13 , and $61[12,13]$. The NRAS mutation at codon 61 accounted for $67-88 \%$ of all RAS mutations [14, 15]. NRAS mutations were more common in conventional FNs than Hürthle-cell neoplasms (HCNs) [14]. However, the diagnostic importance of RAS mutations has 


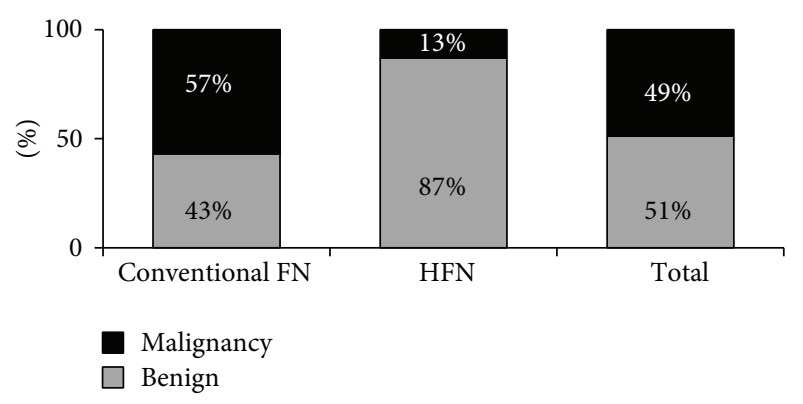

(a)

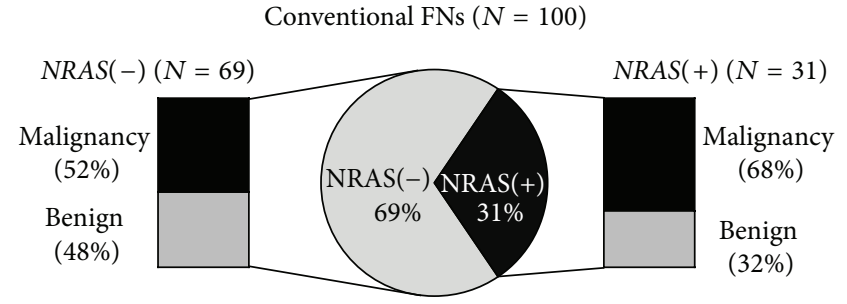

(b)

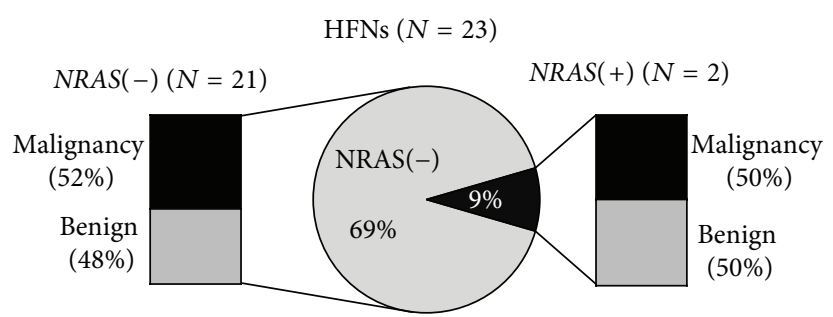

(c)

FIgURE 1: (a) Postoperative surgical pathology results of 123 thyroid nodules with a preoperative diagnosis of follicular neoplasm (FN) according to the histological subtypes. (b) Surgical results and NRAS mutation status of the 100 conventional FNs. (c) Surgical results and NRAS mutation status of the 23 Hürthle cell neoplasms (HFNs).

been ambiguous because these mutations are found not only in thyroid cancers but also in histologically benign nodules, including follicular adenomas and nodular hyperplasia [15].

In the present study, we analyzed the clinical significance and diagnostic utility of NRAS mutations in patients who underwent surgery for FN.

\section{Subjects and Methods}

2.1. Patient Population and Inclusion Criteria. This study was performed under Institutional Review Board approval at Seoul St. Mary's Hospital, the Catholic University of Korea. We analyzed the prospectively collected records associated with 123 patients who had a preoperative diagnosis of FN and then underwent surgery from 2012 to 2013 . FNAB $(n=$ 47 ) or $\mathrm{CNB}(n=76)$ was performed preoperatively to diagnose the thyroid nodules. When thyroid surgery was indicated, a diagnostic lobectomy and isthmusectomy were recommended. A completion thyroidectomy was offered to patients with a surgically proven FTC with vascular invasion regardless of tumor size or Hürthle cell carcinoma after detailed counseling. All samples were rereviewed by a pathologist who specializes in thyroid pathology (CKJ). The formal diagnosis was made according to the World Health Organization classification of thyroid cancer.

2.2. NRAS Mutation Analysis. DNA was extracted from unstained paraffin-embedded tissue sections of surgical resection specimens using the QIAamp DNA FFPE Tissue Kit (Qiagen, Hilden, Germany) after manual microdissection. After PCR using primers targeting codon 61 using the PyroMark PCR Kit (Qiagen), pyrosequencing was performed according to standard procedures using the NRAS Pyro Kit (Qiagen) and PyroMark Q24 System (Qiagen). Program outputs were analyzed with the PyroMark Q24 software (version 2.0.6; Qiagen) using allele quantification mode. The NRAS mutation status was considered positive if the mutant allele percentage was $5 \%$ or more.

2.3. Statistical Analysis. Statistical analyses were performed with the SPSS software (version 18.0; SPSS, Chicago, IL, USA). The $\chi^{2}$ test was used to compare proportions between categorical variables and the $t$-test was used to compare means between groups. Probability values $<0.05$ were considered to indicate statistical significance.

\section{Results}

3.1. Surgical Results of FNs. In total, the 123 FNs consisted of 100 conventional and $23 \mathrm{HCN}$ subtypes (Figure 1). Malignancy was histologically proven in 57 of 100 conventional FNs and 3 of 23 HCNs. The overall malignancy rate was $48.8 \%$ (60/123 FNs). The malignancy rate was higher in conventional FN than in HCN (57\% versus $13 \%$; $P<0.001$ ). Of $123 \mathrm{FNs,}$ 33 harbored NRAS mutations. The mutation rate was higher in conventional FN than in $\mathrm{HCN}$ (31\% versus 9\%; $P=0.029$ ) (Figure 1).

3.2. Clinicopathological Features and NRAS Mutation Status of FNs. Of 123 FNs, 33 (26.8\%) harbored NRAS mutations. NRAS Q61R (c.182A >G) mutants were found in 26 cases. Seven FNs harbored the NRAS Q61K (c.181C>A) mutation (Figure 2). The mutation rate was higher in conventional FN than in $\mathrm{HCN}$ (31\% versus $9 \% ; P=0.029)$. The frequency 


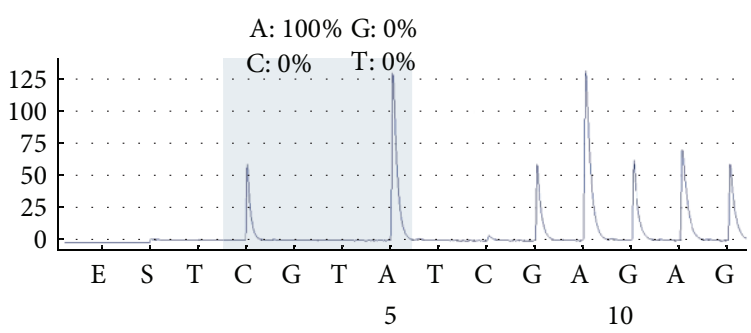

(a)

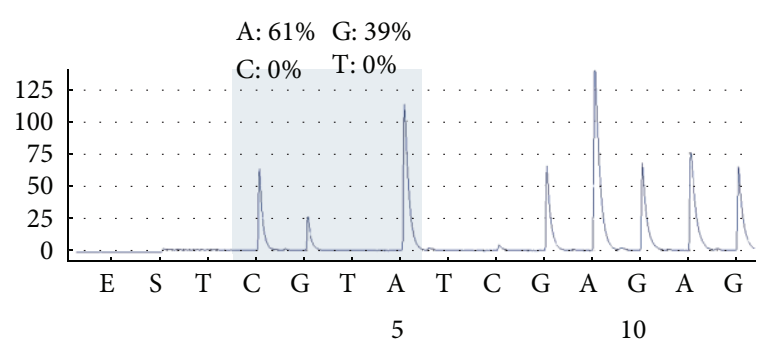

(b)

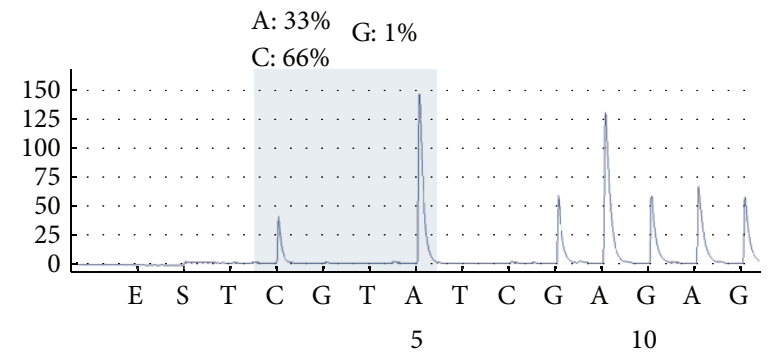

(c)

FIGURE 2: Analysis of NRAS mutation by pyrosequencing. Representative images of wild type (a), NRAS Q61R (c.182A>G) (b), and NRAS Q61K (c.181C>A) (c).

of NRAS mutation according to the histological subtype of FN is summarized in Figure 1. The age of patients with FN ranged from 16 to 83 years (mean $48.7 \pm 14.1$ ). Age and gender were not significantly correlated with the NRAS mutation status (Table 1). Tumor size was larger in FNs without NRAS mutations $(P<0.001)$ (Table 1$)$.

3.3. Risk of Malignancy in Patients with a NRAS MutationPositive FN. NRAS mutations were found more frequently in malignant than in benign nodules $(36.7 \%$ versus $17.5 \%$; $P=0.016)$. The sensitivity, specificity, positive predictive value, and negative predictive value of a NRAS mutationpositive FN specimen to predict malignancy were $37 \%, 83 \%$, $67 \%$, and $58 \%$, respectively. Of all 123 patients, 60 had one or more additional thyroid nodules in addition to FN. Among the 60 patients with additional nodules, 24 had malignancy diagnosed incidentally during FN treatment. Finally, 73 of 123 patients had malignant nodules after surgery. There was a tendency that the patients with incidental malignancy in addition to FN had a higher rate of NRAS mutation in their FN than those with additional benign nodules $(50 \%$ versus $28 \%$; $P=0.080$; Table 1 ). When we considered patients with a malignant nodule diagnosed from $\mathrm{FN}$ and/or another nodule as finally having thyroid malignancy, 26 of 33 (79\%) patients with a NRAS-positive FN had thyroid malignancy whereas 47 of $90(52 \%)$ patients with a NRAS-negative FN had malignancy (Table 1).

3.4. Subanalysis of 60 Patients Having Histologically Proven Thyroid Cancers. Of a total of 123 patients with a preoperative diagnosis of FN, 60 were proven to have malignant FN after thyroid surgery. We conducted a subanalysis of 60 thyroid cancer patients to examine the clinicopathologic significance of NRAS mutation in thyroid cancer (Table 2). The age of the thyroid cancer patients ranged from 24 to 81 years ( 38 females, 22 males). The 60 thyroid cancers consisted of 37 (62\%) encapsulated follicular variant of papillary thyroid cancers (FVPTCs), 6 (10\%) infiltrative FVPTCs, 3 (5\%) classic PTCs, 13 (22\%) FTCs, one widely invasive, 12 minimally invasive tumors, and $1(2 \%)$ poorly differentiated carcinoma. Histological evaluations revealed the invasion of tumor capsules in 34 tumors and vascular invasion in nine tumors. No significant difference was observed in the frequency of NRAS mutations in terms of capsular invasion or vascular invasion. Tumor size was significantly larger in cancers without NRAS mutations than in those with mutations $(P=0.012$; Table 2$)$.

\subsection{Subanalysis of 63 Patients with Histologically Proven} Benign Thyroid Lesions. Of a total of 123 patients with a preoperative diagnosis of FN, 63 were proven to have benign thyroid lesions after thyroid surgery (Table 3). We further analyzed the relationship between NRAS mutation and clinicopathological features in 63 patients with benign nodules. The age of patients with benign thyroid lesions ranged from 16 to 83 ( 54 females, 9 males). The 63 benign thyroid lesions included 21 follicular adenomas and 12 nodular hyperplasias. Age and gender did not correlate with NRAS mutation status. NRAS-mutated benign tumors had significantly smaller tumor size than benign lesions without NRAS mutations $(P=$ 0.010; Table 3).

3.6. NRAS Mutation Allele Frequency. All NRAS mutations found in the tumor samples were heterozygous somatic mutations. The NRAS mutation allele frequency ranged from $14 \%$ to $46 \%$ (median $33 \%$ ), corresponding to $28-92 \%$ of cells with a heterozygous mutation. There was no difference 
TABLE 1: Clinicopathological features and the NRAS mutation status of 123 follicular neoplasms (FNs).

\begin{tabular}{lcc}
\hline & $\begin{array}{c}\text { Patients with a NRAS } \\
\text { negative } \\
\text { FN }(N=90)\end{array}$ & $\begin{array}{c}\text { Patients with a NRAS } \\
\text { positive }\end{array}$ \\
FN $(N=33)$
\end{tabular}

${ }^{*}$ Of all 123 patients, 60 had one or more incidental nodules in addition to index nodule with a preoperative diagnosis of FN.

TABLE 2: Clinicopathologic features and the NRAS mutation status of 60 thyroid cancers with a preoperative diagnosis of follicular neoplasm.

\begin{tabular}{|c|c|c|c|}
\hline & $\begin{array}{l}\text { Patients with a NRAS } \\
\text { negative thyroid cancer } \\
(N=38)\end{array}$ & $\begin{array}{l}\text { Patients with a NRAS } \\
\text { positive thyroid cancer } \\
\qquad(N=22)\end{array}$ & $P$-value \\
\hline Average tumor size $(\mathrm{cm})$ & $2.2 \pm 1.4$ & $1.4 \pm 0.9$ & 0.012 \\
\hline \multicolumn{4}{|l|}{ Age } \\
\hline$\leq 45$ years & $17(59 \%)$ & $12(41 \%)$ & \multirow{2}{*}{0.464} \\
\hline$>45$ years & $21(68 \%)$ & $10(32 \%)$ & \\
\hline \multicolumn{4}{|l|}{ Gender } \\
\hline Female & $26(62 \%)$ & $16(38 \%)$ & \multirow[t]{2}{*}{0.726} \\
\hline Male & $12(67 \%)$ & $6(33 \%)$ & \\
\hline \multicolumn{4}{|l|}{ Histopathology } \\
\hline Encapsulated FVPTC & $19(51 \%)$ & $18(49 \%)$ & \\
\hline Infiltrative FVPTC & $4(67 \%)$ & $2(33 \%)$ & \\
\hline Classic PTC & $3(100 \%)$ & 0 & \\
\hline FTC & $11(85 \%)$ & $2(15 \%)$ & \\
\hline Poorly differentiated & $1(100 \%)$ & 0 & \\
\hline \multicolumn{4}{|l|}{ Capsular invasion } \\
\hline Negative & $13(50 \%)$ & $13(50 \%)$ & \multirow{2}{*}{0.061} \\
\hline Positive & $25(74 \%)$ & $9(26 \%)$ & \\
\hline \multicolumn{4}{|l|}{ Vascular invasion } \\
\hline Negative & $31(61 \%)$ & $20(39 \%)$ & \multirow{2}{*}{0.329} \\
\hline Positive & $7(78 \%)$ & $2(22 \%)$ & \\
\hline \multicolumn{4}{|l|}{ Lymph node metastasis } \\
\hline Negative & $25(66 \%)$ & $13(34 \%)$ & \multirow{2}{*}{0.400} \\
\hline Positive & $4(50 \%)$ & $4(50 \%)$ & \\
\hline
\end{tabular}

FVPTC, follicular variant of papillary thyroid carcinoma; PTC, papillary thyroid carcinoma; FTC, follicular thyroid carcinoma. 
TABLE 3: Clinicopathologic features and the NRAS mutation status of 63 benign lesions with a preoperative diagnosis of follicular neoplasm.

\begin{tabular}{|c|c|c|c|}
\hline & $\begin{array}{l}\text { Patients with a NRAS } \\
\text { negative benign nodule } \\
\quad(N=52)\end{array}$ & $\begin{array}{l}\text { Patients with a NRAS } \\
\text { positive benign nodule } \\
\qquad(N=11)\end{array}$ & $P$-value \\
\hline Average tumor size $(\mathrm{cm})$ & $2.2 \pm 1.5$ & $1.4 \pm 0.7$ & 0.010 \\
\hline \multicolumn{4}{|l|}{ Age } \\
\hline$\leq 45$ years & $14(74 \%)$ & $5(26 \%)$ & \multirow{2}{*}{0.224} \\
\hline$>45$ years & $38(86 \%)$ & $6(14 \%)$ & \\
\hline \multicolumn{4}{|l|}{ Gender } \\
\hline Female & $45(83 \%)$ & $9(17 \%)$ & \multirow{2}{*}{0.684} \\
\hline Male & $7(78 \%)$ & $2(22 \%)$ & \\
\hline \multicolumn{4}{|l|}{ Histopathology } \\
\hline Follicular adenoma & $41(80 \%)$ & $10(20 \%)$ & \multirow{2}{*}{0.355} \\
\hline Nodular hyperplasia & $11(92 \%)$ & $1(8 \%)$ & \\
\hline
\end{tabular}

in NRAS mutation allele frequencies between benign and malignant tumors (data not shown).

\section{Discussion}

We found that the rate of confirmed malignancy was $48.8 \%$ after surgery of the FNs and FVPTC was the most common type among the malignancies. The NRAS mutation rate was $26.8 \%$ in all FNs. NRAS mutations were more frequent in malignant FNs. Positive and negative predictive values of NRAS mutation testing in FNs for predicting malignancy were $67 \%$ and $58 \%$, respectively. These results were consistent with those of previous studies $[8,15,16]$. Interestingly, the rate of malignancy in additional nodules coexisting with a FN was higher in patients with a NRAS mutation-positive FN than in those with a NRAS mutation-negative FN. Our results showed that overall malignancy rate in patients with a NRAS mutation-positive FN was $78.8 \%$. Gupta et al. found that the presence of RAS mutations in thyroid FNAB specimens was related with an increased incidence of bilateral thyroid cancer and suggested that total thyroidectomy in patients with a RAS mutation-positive nodule is a more appropriate surgical option [17].

We observed that most thyroid cancers positive for NRAS mutation were encapsulated FVPTC and 2 (15\%) of 13 FTCs carried the mutation (Table 2). These findings are consistent with a recent study which found that 13 of 14 NRAS-positive thyroid cancers were FVPTC and one of them was FTC [18]. Pathologic diagnosis of follicular adenoma and FTC essentially requires the lack of nuclear features of PTC. PTC shows nuclear clearing, irregularity, grooves and pseudoinclusions, but the nuclei of follicular adenoma and FTC are round, regular, and not clear. Even so, the differential diagnosis of follicular adenoma and FTC from encapsulated FVPTC can be difficult even for experienced pathologists [19, 20]. The diagnostic threshold for FVPTC has been lowered over the past two decades $[19,20]$. The evolution of criteria that more broadly define the pathologic diagnosis of PTC is partly responsible for a higher proportion of FVPTC rather than FTC diagnosed in our study.
Few studies have examined the role of NRAS mutation as a prognostic factor in FN. Jang et al. recently reported that NRAS mutations at codon 61 mutation were associated significantly with the presence of a distant metastasis in FTC [21]. Fukahori et al. also found that NRAS codon 61 mutations in 58 FTC patients were related with distant metastasis but not with recurrence or overall survival [16]. Volante et al. suggested that RAS mutations were associated with shorter overall survival rate in poorly differentiated cancers [10].

Our study showed that encapsulated FVPTCs were the most common phenotype in malignant FNs. NRAS mutations were found in $49 \%$ of encapsulated FVPTCs. This rate is similar to the overall RAS mutation frequencies, from $18 \%$ to $48 \%$ in follicular adenomas and from $23 \%$ to $57 \%$ in FTCs $[8,16,22-24]$. However, infiltrative FVPTCs had a lower frequency of NRAS mutations.

We observed that most of the tumor samples ( $80 \%)$ had more than $25 \%$ of NRAS mutant alleles, corresponding to more than $50 \%$ of the cells carrying a NRAS heterozygous mutation. Because tumor samples reflect the average degree of all cell types present in the sample, including normal stromal cells, endothelial cells, and inflammatory cells, the finding that the NRAS mutation occurred in more than $50 \%$ of cells within the tumor suggests strongly that it is a clonal mutation in these tumors. In the remaining $20 \%$ of the FNs, the frequency of mutant alleles was $14-24 \%$. One study reported that these tumors could be classified as clonal neoplasms despite the different histological appearance and that many RAS-positive clonal tumors have been misclassified as histologically nonclonal tumors [17].

In our study, there was no difference in the malignancy rate between FNs diagnosed by $\mathrm{CNB}$ and FNAB. The overall malignancy rate of FN and ratio of conventional FN to $\mathrm{HCN}$ were consistent with those of our previous study [25].

Our study showed that FNs with NRAS mutations had no significant differences in clinicopathological features except tumor size compared with NRAS-negative tumors. The presence of NRAS mutations was inversely correlated with tumor size, regardless of the presence of malignancy. This finding differs from those of previous studies [15, 26]. This discrepancy might have been caused by the small number of 
cases and different histological types of tumors in individual series. Further studies across a larger number of cases are needed.

Limitations of the present study include the relatively small sample size and short follow-up period. Surgical specimens were used to test NRAS mutation rather than preoperative FNA or CNB specimens. However, most tumors showed a high mutant allele frequency and results of NRAS mutation in all CNB specimens were consistent with those in surgical specimens. As NRAS mutations occur most frequently in FN and were detected exclusively at codon 61 in exon 3, we only examined the impact of the NRAS codon 61 testing on clinicopathological significance. Further studies are needed to clarify the preoperative role of NRAS mutations for guiding the surgical approach and their prognostic role in patients with FN.

In conclusion, determining NRAS mutation status in FNs is expected to further improve the accuracy of cancer diagnoses and also to help predict cancer risk in thyroid nodules accompanying FNs.

\section{Conflict of Interests}

The authors declare that there is no conflict of interests regarding the publication of this paper.

\section{Acknowledgment}

This research was supported by the Basic Science Research Program through the National Research Foundation of Korea (NRF) funded by the Ministry of Science, ICT and future planning (2013R1A2A2A01068570).

\section{References}

[1] M. Bongiovanni, A. Spitale, W. C. Faquin, L. Mazzucchelli, and Z. W. Baloch, "The Bethesda system for reporting thyroid cytopathology: a meta-analysis," Acta Cytologica, vol. 56, no. 4, pp. 333-339, 2012.

[2] Z. W. Baloch, V. A. LiVolsi, S. L. Asa et al., "Diagnostic terminology and morphologic criteria for cytologic diagnosis of thyroid lesions: a synopsis of the national cancer institute thyroid fine-needle aspiration state of the science conference," Diagnostic Cytopathology, vol. 36, no. 6, pp. 425-437, 2008.

[3] C. Yoo, H. J. Choi, S. Im et al., "Fine needle aspiration cytology of thyroid follicular neoplasm: cytohistologic correlation and accuracy," Korean Journal of Pathology, vol. 47, no. 1, pp. 61-66, 2013.

[4] Y. E. Nikiforov, N. P. Ohori, S. P. Hodak et al., "Impact of mutational testing on the diagnosis and management of patients with cytologically indeterminate thyroid nodules: a prospective analysis of 1056 FNA samples," The Journal of Clinical Endocrinology \& Metabolism, vol. 96, no. 11, pp. 33903397, 2011.

[5] T. Y. Kim, W. B. Kim, J. Y. Song et al., "The $B R A F^{V 600 E}$ mutation is not associated with poor prognostic factors in Korean patients with conventional papillary thyroid microcarcinoma," Clinical Endocrinology, vol. 63, no. 5, pp. 588-593, 2005.
[6] T. Fukushima, S. Suzuki, M. Mashiko et al., "BRAF mutations in papillary carcinomas of the thyroid," Oncogene, vol. 22, no. 41, pp. 6455-6457, 2003.

[7] U. Cho, W. J. Oh, J. S. Bae et al., "Clinicopathological features of rare BRAF mutations in Korean thyroid cancer patients," Journal of Korean Medical Science, vol. 29, no. 8, pp. 1054-1060, 2014.

[8] M. N. Nikiforova, R. A. Lynch, P. W. Biddinger et al., "RAS point mutations and PAX8-PPAR $\gamma$ rearrangement in thyroid tumors: evidence for distinct molecular pathways in thyroid follicular carcinoma," The Journal of Clinical Endocrinology and Metabolism, vol. 88, no. 5, pp. 2318-2326, 2003.

[9] Y. E. Nikiforov, "Thyroid carcinoma: Molecular pathways and therapeutic targets," Modern Pathology, vol. 21, supplement 2, pp. S37-S43, 2008.

[10] M. Volante, I. Rapa, M. Gandhi et al., "RAS mutations are the predominant molecular alteration in poorly differentiated thyroid carcinomas and bear prognostic impact," The Journal of Clinical Endocrinology and Metabolism, vol. 94, no. 12, pp. 47354741, 2009.

[11] G. Garcia-Rostan, H. Zhao, R. L. Camp et al., "ras Mutations are associated with aggressive tumor phenotypes and poor prognosis in thyroid cancer," Journal of Clinical Oncology, vol. 21, no. 17, pp. 3226-3235, 2003.

[12] J. L. Bos, "ras Oncogenes in human cancer: a review," Cancer Research, vol. 49, no. 17, pp. 4682-4689, 1989.

[13] S. R. Lee, C. K. Jung, T. E. Kim et al., "Molecular genotyping of follicular variant of papillary thyroid carcinoma correlates with diagnostic category of fine-needle aspiration cytology: values of RAS mutation testing," Thyroid, vol. 23, no. 11, pp. 1416-1422, 2013.

[14] R.-T. Liu, C.-Y. Hou, H.-L. You et al., "Selective occurrence of ras mutations in benign and malignant thyroid follicular neoplasms in Taiwan," Thyroid, vol. 14, no. 8, pp. 616-621, 2004.

[15] V. Vasko, M. Ferrand, J. di Cristofaro, P. Carayon, J. F. Henry, and C. De Micco, "Specific pattern of RAS oncogene mutations in follicular thyroid tumors," Journal of Clinical Endocrinology and Metabolism, vol. 88, no. 6, pp. 2745-2752, 2003.

[16] M. Fukahori, A. Yoshida, H. Hayashi et al., "The associations between ras mutations and clinical characteristics in follicular thyroid tumors: new insights from a single center and a large patient cohort," Thyroid, vol. 22, no. 7, pp. 683-689, 2012.

[17] N. Gupta, A. K. Dasyam, S. E. Carty et al., "RAS mutations in thyroid FNA specimens are highly predictive of predominantly low-risk follicular-pattern cancers," The Journal of Clinical Endocrinology and Metabolism, vol. 98, no. 5, pp. E914-E922, 2013.

[18] J. H. An, K. H. Song, S. K. Kim et al., "RAS mutations in indeterminate thyroid nodules are predictive of the follicular variant of papillary thyroid carcinoma," Clinical Endocrinology, 2014.

[19] S. Widder, K. Guggisberg, M. Khalil, and J. L. Pasieka, "A pathologic re-review of follicular thyroid neoplasms: the impact of changing the threshold for the diagnosis of the follicular variant of papillary thyroid carcinoma," Surgery, vol. 144, no. 1, pp. 80-85, 2008.

[20] M. J. Armstrong, H. Yang, L. Yip et al., "PAX8/PPARgamma rearrangement in thyroid nodules predicts follicular-pattern carcinomas, in particular the encapsulated follicular variant of papillary carcinoma," Thyroid, 2014. 
[21] E. K. Jang, D. E. Song, S. Y. Sim et al., "NRAS codon 61 mutation is associated with distant metastasis in patients with follicular thyroid carcinoma," Thyroid, vol. 24, no. 8, pp. 1275-1281, 2014.

[22] C. T. Esapa, S. J. Johnson, P. Kendall-Taylor, T. W. J. Lennard, and P. E. Harris, "Prevalence of Ras mutations in thyroid neoplasia," Clinical Endocrinology, vol. 50, no. 4, pp. 529-535, 1999.

[23] G. Manenti, S. Pilotti, F. C. Re, G. D. Porta, and M. A. Pierotti, "Selective activation of ras oncogenes in follicular and undifferentiated thyroid carcinomas," European Journal of Cancer, vol. 30, no. 7, pp. 987-993, 1994.

[24] A. Proietti, C. Sartori, N. Borrelli et al., "Follicular-derived neoplasms: morphometric and genetic differences," Journal of Endocrinological Investigation, vol. 36, no. 11, pp. 1055-1061, 2013.

[25] H. S. Min, J. H. Kim, I. Ryoo, S. L. Jung, and C. K. Jung, "The role of core needle biopsy in the preoperative diagnosis of follicular neoplasm of the thyroid," APMIS: Acta Pathologica, Microbiologica, et Immunologica Scandinavica, 2014.

[26] J. Y. Park, W. Y. Kim, T. S. Hwang et al., "BRAF and RAS mutations in follicular variants of papillary thyroid carcinoma," Endocrine Pathology, vol. 24, no. 2, pp. 69-76, 2013. 


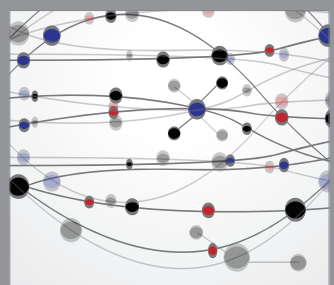

The Scientific World Journal
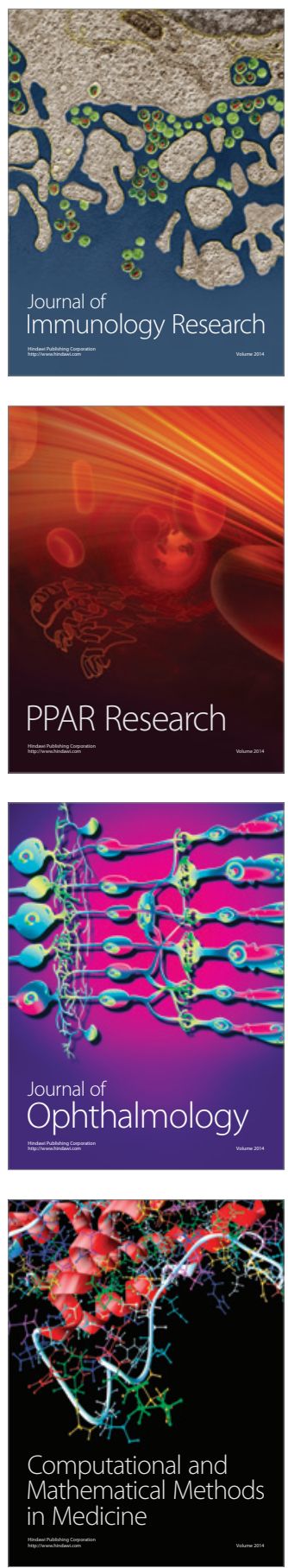

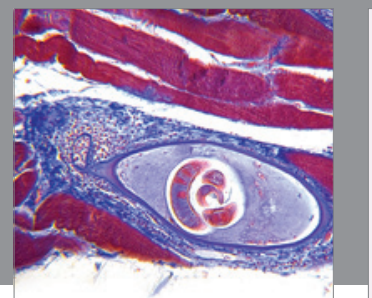

Gastroenterology

Research and Practice
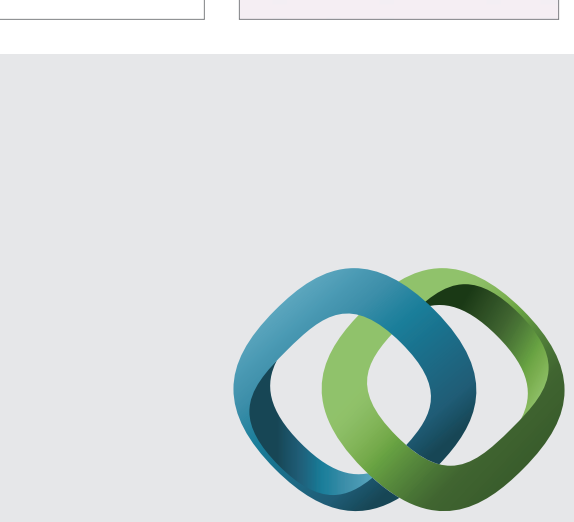

\section{Hindawi}

Submit your manuscripts at

http://www.hindawi.com
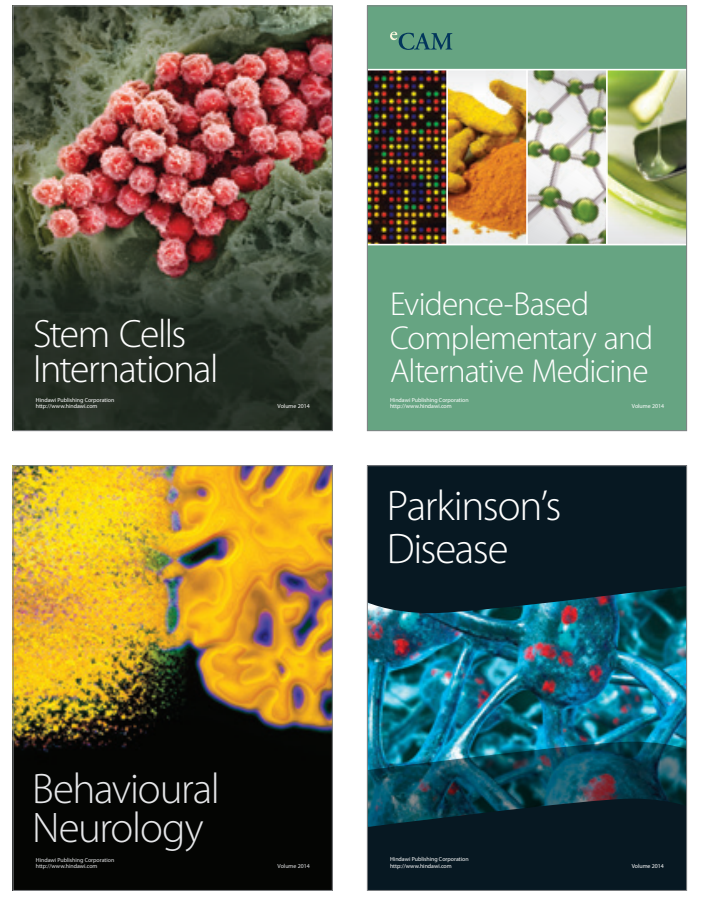
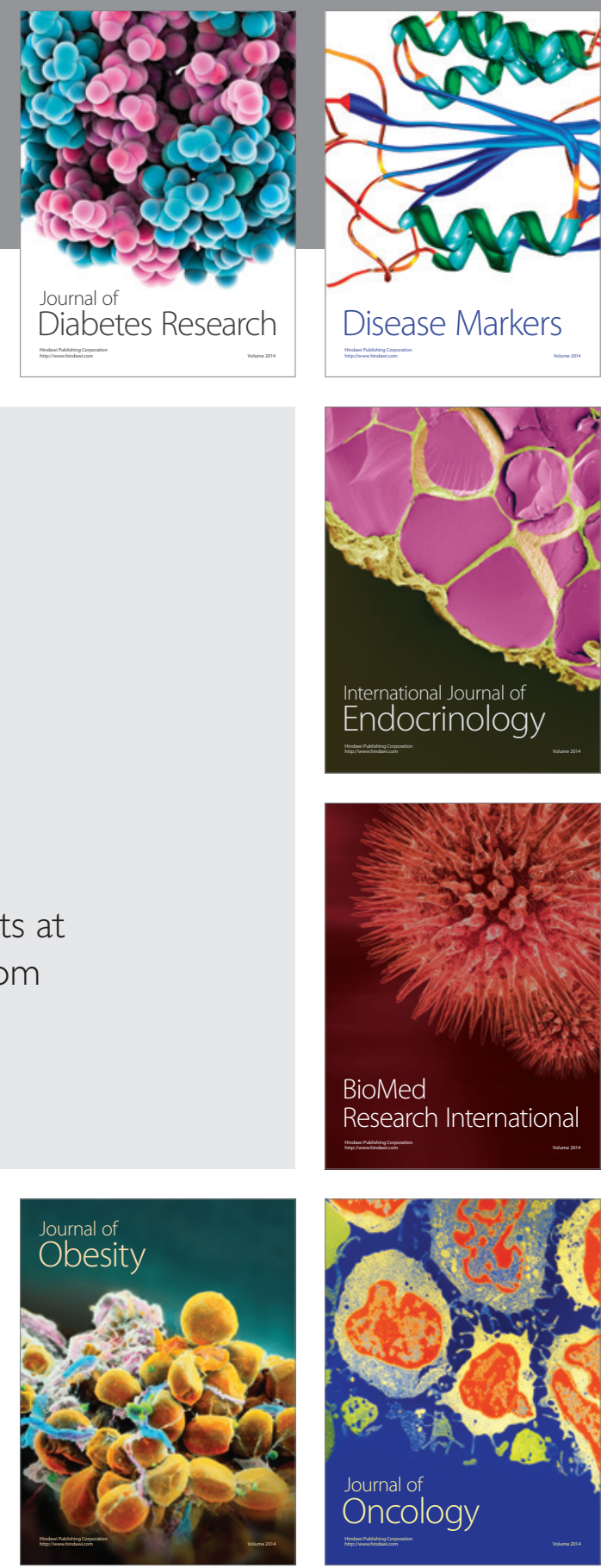

Disease Markers
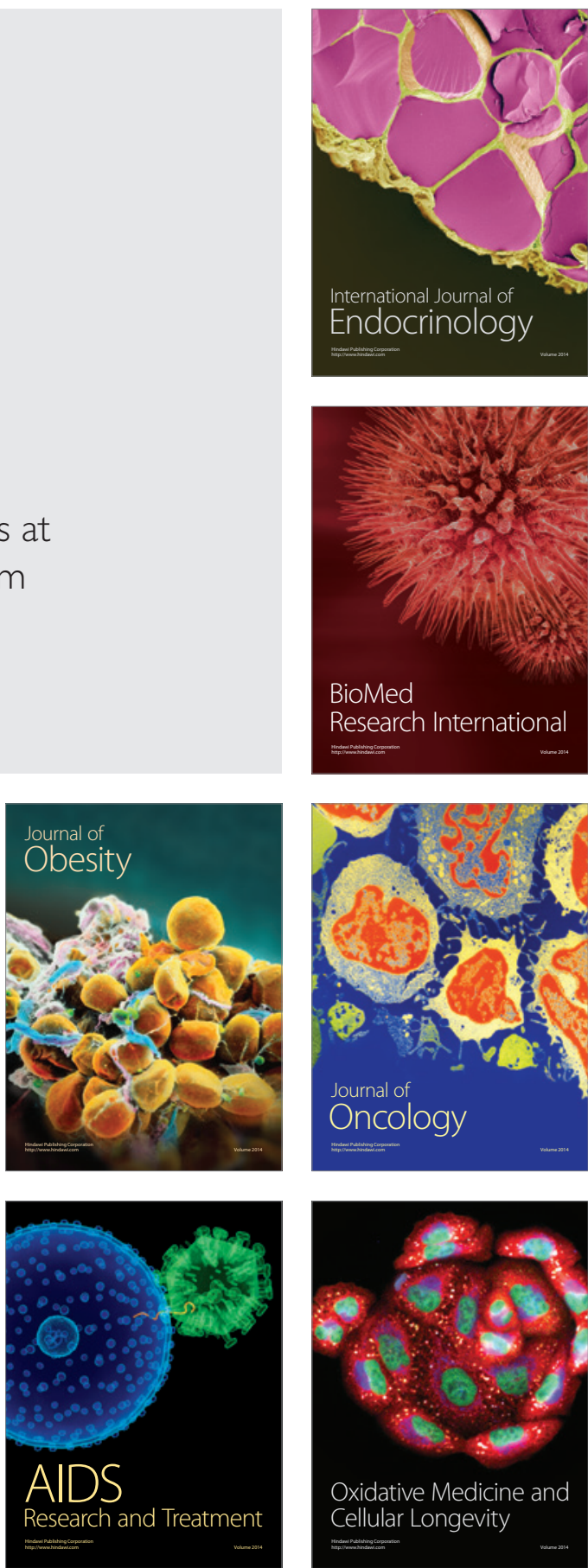